DOI: https://doi.org/10.46296/ig.v4i8.0031

\title{
PRODUCCIÓN DE ETANOL CELULÓSICO A PARTIR DE LA CONVERSIÓN DE RESIDUOS AGRÍCOLAS
}

\section{CELLULOSIC ETHANOL PRODUCTION FROM THE CONVERSION OF AGRICULTURAL WASTE}

\author{
Monroy-Intriago Luigi1*; Zambrano-Zambrano María ${ }^{1}$; Latorre-Castro Gisela ${ }^{1}$; Rosero- \\ Delgado Ernesto ${ }^{1}$ \\ ${ }^{1}$ Carrera de Ingeniería Química, Facultad de Ciencias Matemáticas, Físicas y \\ Químicas, Universidad Técnica de Manabí, UTM. Portoviejo, Ecuador.
}

*Correo: Imonroy5575@utm.edu.ec

\begin{abstract}
Resumen
Los residuos agrícolas constituyen una fuente abundante de material lignocelulósico. Según su conversión física, química o biológica, el material lignocelulósico permite la obtención de etanol. Ésta es una solución al problema de la eliminación final de residuos celulósicos. El objetivo de este trabajo radicó en la determinación de condiciones adecuadas de pretratamiento (\% de humedad y tamaño de partícula) para residuos agrícolas (maní, fréjol y arroz). Además, se evaluó el efecto de las condiciones básicas de hidrólisis a 2, 3 y $4 \%$ de $\mathrm{NaOH}$ a 25,73 y $121 \stackrel{\circ}{\circ} \mathrm{C}$ durante 1 hora, en relación a la cantidad de azúcares reductores. Como resultado, la temperatura influye significativamente en la extracción de azúcares reductores. La fermentación alcohólica de cáscara de maní con S. cerevisiae produjo $4.579 \mathrm{mg}$ de etanol/100 mg de azúcares reductores, en condiciones adecuadas de temperatura alta y concentraciones bajas de $\mathrm{NaOH}$. Este resultado fue comparable al obtenido por otros autores.
\end{abstract}

Palabras clave: residuos agrícolas, conversión, etanol celulósico, azúcares fermentables.

\begin{abstract}
Agricultural residues constitute an abundant source of lignocellulosic material. According to its physical, chemical or biological conversion, lignocellulosic material allows the obtaining of ethanol. This is a solution to the problem of final disposal of cellulosic wastes. The objective of this work was the determination of suitable pretreatment conditions (\% moisture and particle size) for agricultural residues (peanuts, beans, and rice). In addition, the effect of the basic hydrolysis conditions was also evaluated at 2, 3, and $4 \%$ of $\mathrm{NaOH}$ at of 25,73 and $121 \stackrel{\circ}{\circ} \mathrm{C}$ for 1 hour, in relation to the amount of reducing sugars. As a result, the temperature significantly influences the extraction of reducing sugars. The alcoholic fermentation of peanut shell with $S$. cerevisiae yielded $4,579 \mathrm{mg}$ of ethanol / $100 \mathrm{mg}$ of reducing sugars, under suitable conditions of high temperature and low concentrations of $\mathrm{NaOH}$. This result was comparable to that obtained by other authors.
\end{abstract}

Keywords: agricultural residues, conversion, cellulosic ethanol, fermentable sugars.

Información del manuscrito:

Fecha de recepción: 23 de diciembre de 2020

Fecha de aceptación: 25 de enero de 2021

Fecha de publicación: 09 de julio de 2021 


\section{Introducción}

Se estima un incremento en la demanda de energías primarias en todo el mundo de aproximadamente 11,8 millones de toneladas equivalentes de petróleo (Mtep) para el año 2030 (Alam et al., 2013). Se espera que alcance los 18.000 Mtep en el año 2050 (Frei et al., 2013). Los recursos energéticos de mayor demanda son los combustibles fósiles, el carbono y el gas natural, puesto que estos recursos son utilizados para satisfacer el $81,1 \%$ de la demanda energética mundial (Alam et al., 2013). En Ecuador, el consumo de combustibles fósiles representa el $80 \%$ de la demanda energética total (INER, 2016), mientras el $20 \%$ proviene de fuentes de energía renovable como la biomasa, eólica, etc., (Álvarez et al., 2012). Sin embargo, el consumo de los recursos energéticos de origen fósil se considera la principal causa del calentamiento global (Griffiths et al., 2019), debido a la liberación de gases contaminantes como el dióxido de carbono, metano, óxido nitroso, hidrocarburos, material particulado, etc., (Amestoy Alonzo, 2010). En América Latina, el total de emisiones de gases contaminantes es del 15,7\%, del cual los combustibles fósiles aportan el 9,5\%, el carbono el $1,3 \%$ y el gas natural el 4,9\% (Moya et al., 2019; RománCollado \& Morales-Carrión, 2018).

La producción agrícola en Ecuador ha aumentado en los últimos tres años; se registra un incremento del $18 \%$ en la caña de azúcar, y se estima que el arroz se incrementará en un $25 \%$ de toneladas producidas por año, en un período de 3 años (INER, 2016). Ese incremento de producción va relacionado con la acumulación de residuos agrícolas. Por otra parte, Saval (2012) menciona que en la industria cervecera se utiliza apenas el $8 \%$ de los componentes del grano, en la del aceite de palma el 9\%, y en la del café el $9,5 \%$, las cantidades restantes son residuos. En Manabí, la producción agrícola anual del maní (25\% cáscara) es 586 tn, de arroz en cáscara (20\% cáscara) 48.411 th y de fréjol tierno en vaina (30\% cáscara) 802 tn (INEC, 2018). Los residuos agrícolas y agroindustriales se generan en las diferentes etapas del proceso productivo, y representan una problemática a nivel mundial debido a que su inadecuada disposición contribuye a la degeneración 
ambiental (Chávez Porras \&

Rodríguez González, 2016), y al no ser aprovechados se pierde material vegetal potencialmente valioso por sus componentes lignocelulósicos, los cuales podrían ser transformados en productos de elevado valor añadido como los biocombustibles, ya que representan una fuente alternativa de energía renovable (Guerrero et al., 2015).

Los biocombustibles, se obtienen de muchas fuentes orgánicas, entre ellas se encuentra el material lignocelulósico (Sierra et al., 2010). Los residuos agrícolas son materiales lignocelulósicos, los cuales poseen una estructura compleja compuesta de lignina (32$55 \%)$, hemicelulosa (10-34\%) y celulosa (25-67\%) (Rodríguez et al., 2012), son de bajo costo, tienen alto contenido de material fermentable, con un ciclo de combustión de cero emisiones de gases y alta disponibilidad, lo que permite su uso en la producción de biocombustibles como el bioetanol, biodiesel y butanol (Balat, 2011). En Estados Unidos se fomenta la producción y uso del bioetanol producido a partir de maíz, para reactivar el sector agrícola cuando existe sobreproducción de productos agrícolas (Balat \& Balat, 2009); se utiliza como combustible E85 (una mezcla de combustible de $85 \%$ bioetanol y 15\% gasolina) en vehículos diseñados para su uso, aunque E85 representa sólo el 1\% del consumo de bioetanol (Yacobucci \& Schnepf, 2007). En Ecuador, se comercializa el combustible "Ecopaís", siendo el único que contiene derivados de caña de azúcar, está compuesto por un $5 \%$ de bioetanol y un $95 \%$ de gasolina extra con un octanaje de 87; sin embargo, la norma INEN-935, relacionada con derivados de petróleo, dispone incorporar hasta un $15 \%$ de etanol (El Comercio, 2019). Por otra, el uso de la caña de azúcar como materia prima para la producción de bioetanol, ha generado preocupación por un posible incremento del coste de la caña de azúcar y sus derivados; debido a esto, productos como maíz, piñón, sorbo, entre otros, también podrían ser útiles en este plan (EI Comercio, 2018). Según Balat (2007) el bioetanol es el biocombustible más utilizado para el transporte a nivel mundial. El bioetanol obtenido a partir de la conversión del material 
lignocelulósico permite reducir el consumo de combustibles fósiles y la degeneración ambiental (Balat \& Balat, 2009). En base a la necesidad de plantear nuevas alternativas energéticas que mejoren la calidad del medio ambiente y a la vez, la calidad de vida de las personas y sin afectar al sector alimenticio y productivo, esta investigación tiene como objetivo establecer las condiciones más adecuadas para la hidrólisis de residuos agrícolas que permitan alcanzar la mayor conversión de azúcares reductores $(A z R)$, en función de la producción de bioetanol o etanol celulósico.

\section{Metodología}

\subsection{Residuos agrícolas}

Se utilizaron los residuos de los productos agrícolas más producidos en la provincia de Manabí. Las cáscaras de Maní, Fréjol y la cascarilla de Arroz, se obtuvieron de cultivos temporales del cantón Rocafuerte, Manabí, Ecuador.

\subsection{Acondicionamiento de los residuos agrícolas}

El acondicionamiento inicial, consistió en determinar el porcentaje de humedad, así como estandarizar el tamaño de partícula $(\varnothing=1 \mathrm{~mm})$ de los residuos agrícolas por molienda, para posteriormente ser almacenados en recipientes plásticos herméticos a temperatura ambiente. La humedad fue determinada con una termo balanza (Boenco) a $100^{\circ} \mathrm{C}$, el resultado se expresó como \% humedad por gramo de residuo (Torres Robles et al., 2018).

\subsection{Pretratamiento básico}

El pretratamiento básico tuvo el objetivo de disociar la celulosa y hemicelulosa en azúcares fermentables y consistió en incorporar en matraces de $50 \mathrm{~mL}, 5$ gramos del residuo agrícola con $\mathrm{NaOH}$ diluido (2, 3 y 4\%) en relación 1:8 (m/v). Estos fueron sometidos a 3 niveles de temperatura $(25,73$ y $\left.121^{\circ} \mathrm{C}\right)$, por una hora. Una vez finalizado el pretratamiento, se filtró el hidrolizado con papel filtro $(\varnothing=20 \mu \mathrm{m})$, recuperando la fase líquida de interés, y posteriormente se determinó la concentración de AzR (Rodríguez et al., 2017).

\subsection{Contenido de azúcares reductores}

La determinación de los AzR se realizó con la metodología descrita por Miller (1959); la cual consiste en 
la conversión del ácido 3,5 dinitrosalić́lico a ácido 3-amino-5dinitrosalićlico (reactivo que absorbe energía ultravioleta a 540 $\mathrm{nm}$ ) en presencia de AzR.

\subsection{Técnica del DNS}

Se mezclaron $0,5 \mathrm{~mL}$ de residuo y $0,5 \mathrm{~mL}$ del reactivo DNS (3,5Dinitrosalicylic acid) en tubos falcón (Nest) de $15 \mathrm{~mL}$, con la finalidad de detectar los AzR presentes. La reacción se realizó en baño María, con la ayuda de una placa calefactora (Thermo, USA), a $90 \stackrel{\circ}{\circ}$ durante 5 minutos. Luego, se enfrió hasta temperatura ambiente y se añadieron $5 \mathrm{~mL}$ de agua destilada a cada muestra. Los tubos se llevaron a agitación (Pulsing Vortex Mixer, Fisher Scientific) y se realizó la lectura de la absorbancia a $540 \mathrm{~nm}$ (Thermo Scientific GENESYS 10SSpectrophotometers) (Bello Gil et al., 2006). La muestra del hidrolizado debe tener un $\mathrm{pH}$ (Accumet ${ }^{\circledR}$, Fisher Scientific) no menor a 4 para realizar la medición correcta de AzR en el espectrofotómetro, de acuerdo con la técnica citada por Loboguerrero (2007).

\subsection{Fermentación líquida}

Se realizó la hidrólisis del residuo por triplicado, utilizando la concentración de $\mathrm{NaOH}$ y temperatura más adecuada, establecidas conforme al literal "C" de esta sección, con el objetivo de obtener AzR. Se hidrolizaron 20 gramos de cáscara de Maní en relación 1:8 con $\mathrm{NaOH}$ al $2 \%$. Previo a la fermentación se ajustó el pH de los hidrolizados a un valor entre 5 a 6 ; posteriormente, se llevó a un biorreactor de $500 \mathrm{~mL}$ y se adicionaron $2,5 \% \mathrm{~m} / \mathrm{v}$ de levadura $S$. cerevisiae liofilizada. Una vez iniciado el proceso fermentativo, se tomaron lecturas del consumo inicial de sustrato $(A z R)$.

\subsection{Destilación del etanol celulósico}

La destilación consistió en separar el etanol del mosto fermentado, para esto se tomaron $100 \mathrm{~mL}$ de muestra y utilizando un equipo de destilación y una placa calefactora se llevó a temperatura de ebullición del etanol $\left(78,5^{\circ} \mathrm{C}\right)$, se tomaron muestras del volumen destilado para determinar el contenido en \% alcohol (Nzelibe \& Okafoagu, 2007). 


\subsection{Contenido del etanol celulósico}

La concentración de etanol $(\% \mathrm{~V} / \mathrm{V})$ en las muestras del destilado, se cuantificó mediante un refractómetro de alcohol modelo RHV-80ATC (Gradindex, USA). Para calcular el bioetanol producido, se multiplica el volumen de etanol destilado por el \% de alcohol medido con el refractómetro, y se obtiene el etanol producido en $\mathrm{mL}$, luego se multiplica el volumen de etanol por la densidad $(0,8033 \mathrm{~g} / \mathrm{mL})$ y se obtiene el etanol producido en g. (Bakare et al., 2019).

\section{9 .}

Rendimientos

\section{estequiométricos}

Para el cálculo de los rendimientos teóricos del proceso de fermentación, se utilizó la metodología descrita por Doran (1998), en la que se utiliza la ecuación estequiométrica del proceso de fermentación de células heterótrofas (Ec.1).

$$
\begin{aligned}
& C_{w} H_{x} O_{y} N_{z}+b N H_{i} \rightarrow c C H_{\alpha} O_{\beta} N_{\delta}+d C O_{2}+ \\
& e \mathrm{H}_{2} \mathrm{O}+\mathrm{fC}_{\mathrm{j}} \mathrm{H}_{k} \mathrm{O}_{l} \mathrm{~N}_{m} \text { (Ec. } 1 \text { ) } \\
& \mathrm{C}_{\mathrm{w}} \mathrm{H}_{\mathrm{x}} \mathrm{O}_{\mathrm{y}} \mathrm{N}_{\mathrm{z}}=\text { Sustrato } \\
& \mathrm{bNH}_{\mathrm{i}}=\text { Fuente de Nitrógeno } \\
& \mathrm{cCH}_{\alpha} \mathrm{O}_{\beta} \mathrm{N}_{\delta}=\text { Biomasa } \\
& \mathrm{eH}_{2} \mathrm{O}=\text { Agua } \\
& \mathrm{fC}_{\mathrm{j}} \mathrm{H}_{\mathrm{k}} \mathrm{O}_{\mathrm{l}} \mathrm{N}_{\mathrm{m}}=\text { Etanol }
\end{aligned}
$$

Se calcularon los rendimientos máximos estequiométricos, velocidad de consumo de sustrato y generación de producto. Así como cada uno de los coeficientes de la ecuación.

\subsection{Análisis estadístico}

Las variables estudiadas fueron la concentración de $\mathrm{NaOH}$ y la temperatura de hidrólisis en relación a la cantidad de AzR producidos durante el pretratamiento básico, utilizando 3 niveles para la concentración de $\mathrm{NaOH}$ (2, 3, 4 \% $\mathrm{NaOH})$ y la temperatura $(25,73,121$ $\left.{ }^{\circ} \mathrm{C}\right)$, mediante un diseño factorial $3^{2}$. Los valores obtenidos de AzR del proceso de hidrólisis básica, fueron comparados mediante la prueba de múltiples rangos con la ayuda del paquete estadístico Statgraphics Centurion XV.I.

\section{Resultados y discusión}

Los procesos de conversión energética de los residuos lignocelulósicos son afectados por la humedad (Barakat et al., 2015). Para la mayoría de estos procesos, es indispensable que la biomasa tenga un contenido de humedad inferior al 30\% (Escalante Hernández et al., 2010; McKendry, 2002). Por otra 
parte, el tamaño de partícula por medio del proceso de molienda permite aumentar el área de la superficie de hidrólisis (Galbe \& Zacchi, 2007). Por lo tanto, en el presente trabajo, los residuos fueron acondicionados para reducir el contenido de humedad y su tamaño; luego, se realizaron análisis de humedad de los residuos agrícolas (Fig. 1).
A.

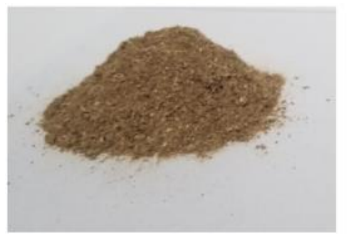

B.

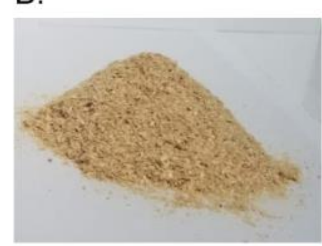

C.

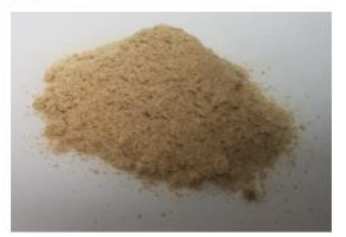

Figura 1. Residuos Agrícolas: (A) Cáscara de Maní, (B) Cáscara de Fréjol, (C) Cascarilla de Arroz

Para la interpretación de los resultados, se realizó la prueba de múltiples rangos y análisis de varianza de los datos en base a los AzR como variable de respuesta en función de la temperatura y concentración de $\mathrm{NaOH}$ durante el proceso de hidrólisis. Los resultados se muestran a continuación en la Tabla 1.

Tabla 1.

Contenido de humedad de los residuos agrícolas

\begin{tabular}{|c|c|}
\hline Material & $\% \mathbf{H}$ \\
\hline Cascarilla de Arroz & 10,09 \\
\hline Cáscara Fréjol & 13,81 \\
\hline Cáscara de Maní & 9,31 \\
\hline
\end{tabular}

$\% \mathrm{H}: \% H u m e d a d$ por gramo de residuo

Se observaron diferencias significativas con respecto a cada parámetro. Sin embargo, es necesario mencionar que, la composición de los compuestos principales (es decir, celulosa, hemicelulosa, lignina) es diferente para cada planta y sus diferentes tipos de pared celular vegetal. La conversión de los materiales lignocelulósicos depende no solo de la composición bioquímica de las 
plantas, sino también de la organización de los tejidos y de cómo está constituida su pared celular (Barakat et al., 2007). Por lo tanto, los residuos analizados no presentan el mismo comportamiento debido a las diferencias de sus características propias (Fig.1).

La temperatura influye en el pretratamiento de material vegetal porque, permite degradar la estructura donde se encuentran almacenadas la celulosa y hemicelulosa.

En la tabla 2 se muestra la cantidad de AzR producida en función de la temperatura; y se observa que las dos variables son directamente proporcionales, es decir, que el aumento de temperatura incrementa la producción de los AzR presentes en los residuos. Este resultado, demuestra que en el tratamiento realizado a temperatura de $121^{\circ} \mathrm{C}$ se produjo una mayor cantidad de AzR en comparación con el tratamiento realizado a 25 y $73^{\circ} \mathrm{C}$, siendo las Cáscaras de Maní (6,12 $\pm 0,8 \mathrm{~g} / \mathrm{L})$ y las Cáscara de Fréjol (4,19 \$ 1,5 g/L) los dos residuos agroindustriales de los cuales se puede obtener la mayor cantidad de AzR en comparación con la Cascarilla de Arroz $(1,77 \pm 0,4$
g/L). Esto fue demostrado por Bastidas (2014), quien menciona que a mayores temperaturas y menores tiempos de residencia se favorece la dilución total de hemicelulosa y parcial de lignina para la formación de AzR. Por otra parte, Barrios Caballero (2014), utilizó los residuos de Maguey peruano, para producción de bioetanol, y pudo demostrar que al aumentar la temperatura gradualmente, durante la hidrólisis, se alcanzó una conversión máxima de 7,31 $\pm 0,05 \mathrm{~g}$ AzR/100 g de muestra a $110^{\circ} \mathrm{C}$ durante 10 horas en autoclave. Sin embargo, Cabrera et al. (2014) describen que los pretratamientos alcalinos y con peróxido alcalino a temperatura suave han demostrado ser métodos factibles en términos de obtención de AzR a partir de paja y cáscara de arroz, aunque se ha demostrado que existe pérdida de sólidos de hasta $35,4 \%$ y $39,3 \%$ para paja y cascarilla de arroz, respectivamente; por lo cual no mejoran los rendimientos de hidrólisis. Las conversiones más altas de azúcares reductores para paja de arroz, se obtuvieron con pretratamientos alcalinos a bajas concentraciones. 
Tabla 2.

Pruebas de múltiples rangos para AzR-residuo en función de la temperatura de hidrólisis

\begin{tabular}{|c|c|c|c|}
\hline \multirow{2}{*}{$\mathbf{T}$} & \multicolumn{3}{|c|}{ Azúcares Reductores (g/L) } \\
\cline { 2 - 4 }$\left({ }^{\circ} \mathbf{C}\right)$ & $\begin{array}{c}\text { Cascarilla de } \\
\text { Arroz }\end{array}$ & $\begin{array}{l}\text { Cáscara } \\
\text { de Fréjol }\end{array}$ & $\begin{array}{c}\text { Cáscara de } \\
\text { Maní }\end{array}$ \\
\hline 25 & $1,07 \pm 0,3^{\mathrm{a}}$ & $2,69 \pm 0,4^{\mathrm{a}}$ & $3,49 \pm 0,7^{\mathrm{a}}$ \\
\hline 73 & $1,09 \pm 0,2^{\mathrm{a}}$ & $3,49 \pm 0,4^{\mathrm{a}}$ & $3,56 \pm 1,5^{\mathrm{a}}$ \\
\hline 121 & $1,77 \pm 0,4^{\mathrm{b}}$ & $4,19 \pm 1,5^{\mathrm{b}}$ & $6,12 \pm 0,8^{\mathrm{b}}$ \\
\hline
\end{tabular}

T: Temperatura

a-b: Los valores de AzR con diferente simbología presentan diferencias significativas (LSD).

En la tabla 3 se indica la concentración de AzR producida en relación a la concentración de $\mathrm{NaOH}$ durante el pretratamiento hidrolítico; se observa que al aumentar la concentración de $\mathrm{NaOH}$, los AzR producidos tienen un incremento poco significativo en los residuos tratados. La Cascarilla de Arroz pretratada con $\mathrm{NaOH}$ al 2\% (1,15 \pm $0,1 \mathrm{~g} / \mathrm{L})$, al $3 \%(1,33 \pm 0,3 \mathrm{~g} / \mathrm{L})$ y al $4 \%(1,4 \pm 0,6 \mathrm{~g} / \mathrm{L})$ indica que hay un ligero incremento de concentración de $A z R$ en relación a la concentración de $\mathrm{NaOH}$, por lo que resulta conveniente el tratamiento con baja concentración de $\mathrm{NaOH}$. Por otra parte, para la Cáscara de Maní $(4,79 \pm 1,4 \mathrm{~g} / \mathrm{L})$ y la Cáscara de Fréjol $(3,85 \pm 0,7 \mathrm{~g} / \mathrm{L})$, resulta ser más conveniente el pretratamiento realizado con $\mathrm{NaOH}$ al $3 \%$, ya que se produce una cantidad de AzR significativamente mayor a las producidas con $\mathrm{NaOH}$ al $2 \%$, y a la vez, no presenta elevadas diferencias significativas en comparación con los $\mathrm{AzR}$ producidos con $\mathrm{NaOH}$ al $4 \%$.

Tabla 3.

Pruebas de múltiples rangos para AzR-residuo en función de la concentración de hidrólisis

\begin{tabular}{|c|c|c|c|}
\hline \multirow{2}{*}{$\mathbf{C}$} & \multicolumn{3}{|c|}{ Azúcares Reductores (g/L) } \\
\cline { 2 - 4 }$(\mathbf{v} / \mathbf{v})$ & $\begin{array}{c}\text { Cascarilla } \\
\text { de Arroz }\end{array}$ & $\begin{array}{c}\text { Cáscara de } \\
\text { Fréjol }\end{array}$ & $\begin{array}{c}\text { Cáscara de } \\
\text { Maní }\end{array}$ \\
\hline 2 & $1,15 \pm 0,1^{\mathrm{a}}$ & $2,62 \pm 1,1^{\mathrm{a}}$ & $3,58 \pm 1,6^{\mathrm{a}}$ \\
\hline
\end{tabular}




\begin{tabular}{|c|c|c|c|}
\hline 3 & $1,33 \pm 0,3^{\mathrm{ab}}$ & $3,85 \pm 0,7^{\mathrm{b}}$ & $4,79 \pm 1,4^{\mathrm{b}}$ \\
\hline 4 & $1,4 \pm 0,6^{\mathrm{b}}$ & $3,89 \pm 1,0^{\mathrm{b}}$ & $4,88 \pm 1,7^{\mathrm{b}}$ \\
\hline
\end{tabular}

C: Concentración de $\mathrm{NaOH}$

a-b: Los valores de AzR con diferente simbología presentan diferencias significativas (LSD).

Sobre esto Michelena et al. (2009), mencionan en su estudio que la concentración de $\mathrm{NaOH}$ durante la hidrólisis del bagazo de caña de azúcar, tiene una influencia poco significativa en relación al porcentaje de celulosa extraída como sustrato para la producción de etanol. Por otra parte Kim \& Han (2012), a partir de análisis estadísticos pudieron determinar que la concentración de $\mathrm{NaOH}$, temperatura de reacción y tiempo de pretratamiento, tienen una influencia significativa sobre el rendimiento de glucosa en la paja de arroz; su rendimiento máximo de glucosa fue de 252,62 $\mathrm{g} / \mathrm{kg}$ de paja de arroz, obtenido en condiciones de $\mathrm{NaOH}$ de $2,96 \%$, a $81,8^{\circ} \mathrm{C}$ durante 56,7 minutos.

\subsection{Fermentación de la Cáscara de Maní}

Las etapas de fermentación y destilación fueron realizadas únicamente a la Cáscara de Maní, ya que es el residuo lignocelulósico, que permitió alcanzar una mayor producción de AzR.

La hidrólisis fue realizada en condiciones de $\mathrm{NaOH}$ al $3 \%$ y $121^{\circ} \mathrm{C}$ por una hora, las cuales se determinaron adecuadas según el análisis comparativo antes realizado. Posteriormente, se evaluó la producción de etanol en relación al tiempo, para esto se tomaron muestras del mosto fermentado y se midieron en el espectrofotómetro cada 30 minutos. El etanol producido se observa en la Fig. 2.

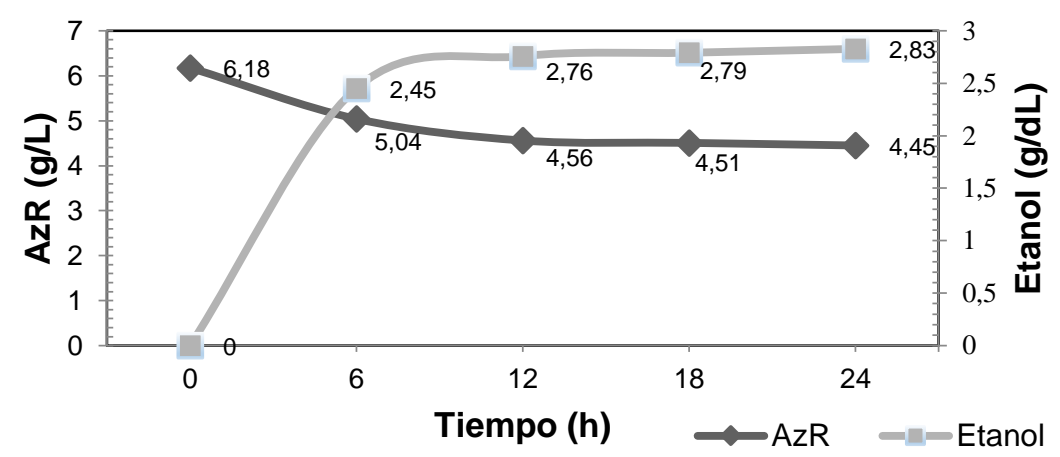

Figura 2. Cinética de Fermentación de la Cáscara de Maní, consumo de AzR y producción de etanol a partir de la cascará de maní. 
3.2. Cálculos de los coeficientes estequiométricos para la fermentación de la Cáscara de Maní

En esta investigación se calcularon los coeficientes estequiométricos en gramos, a partir del rendimiento de etanol producido experimentalmente, y tomando como referencia la composición porcentual de sacarosa, glucosa, xilosa, arabinosa, producidas a partir de la hidrólisis de cascarilla de arroz con $\mathrm{NaOH}$ al $2 \%$ descrita por (Camacho Villanueva et al., 2015).

- Glucosa:

$\mathrm{C}_{6} \mathrm{H}_{12} \mathrm{O}_{6}+0.0873 \mathrm{NH}_{3}$

$\rightarrow 5.1371 \mathrm{C}_{1} \mathrm{H}_{1,83} \mathrm{O}_{0,56} \mathrm{~N}_{0,017}+0.8518 \mathrm{CO}_{2}$

$+1.4139 \mathrm{H}_{2} \mathrm{O}+0.0055 \mathrm{C}_{2} \mathrm{H}_{6} \mathrm{O}_{1}$

- Xilosa:

$\mathrm{C}_{5} \mathrm{H}_{10} \mathrm{O}_{5}+0.0727 \mathrm{NH}_{3}$

$\rightarrow 4.2779 \mathrm{C}_{1} \mathrm{H}_{1,83} \mathrm{O}_{0,56} \mathrm{~N}_{0,017}$

$+0.7105 \mathrm{CO}_{2}+1.1774 \mathrm{H}_{2} \mathrm{O}$

$+0.0057 \mathrm{C}_{2} \mathrm{H}_{6} \mathrm{O}_{1}$

- Arabinosa

$\mathrm{C}_{5} \mathrm{H}_{10} \mathrm{O}_{5}+0.0721 \mathrm{NH}_{3}$

$\rightarrow 4.2421 \mathrm{C}_{1} \mathrm{H}_{1,83} \mathrm{O}_{0,56} \mathrm{~N}_{0,017}$

$+0.7185 \mathrm{CO}_{2}+1.1676 \mathrm{H}_{2} \mathrm{O}$

$+0.0196 \mathrm{H}_{6} \mathrm{O}_{1}$

- Sacarosa

$\mathrm{C}_{12} \mathrm{H}_{22} \mathrm{O}_{11}+0.1744 \mathrm{NH}_{3}$

$\rightarrow 10.2643 \mathrm{C}_{1} \mathrm{H}_{1,83} \mathrm{O}_{0,56} \mathrm{~N}_{0,017}$

$+1.7059 \mathrm{CO}_{2}+1.8252 \mathrm{H}_{2} \mathrm{O}$

$+0.0148 \mathrm{C}_{2} \mathrm{H}_{6} \mathrm{O}_{1}$
El rendimiento obtenido a partir de la fermentación de la cáscara de maní fue de $4,579 \mathrm{mg}$ de etanol /100 mg de $A z R$, mostrando valores similares al trabajo realizado por Piñeros \& Otálvaro (2010), el cual obtuvo un rendimiento de $2,87 \mathrm{mg}$ de etanol /100 mg de cascarilla de Arroz, a partir de un tratamiento alcalinoenzimático. Por otra parte, Cacua et al. (2018) realizaron el pretratamiento básico de la cascarilla de arroz obteniendo un rendimiento de 7,778 $\mathrm{g} / \mathrm{L}$ de $A z R$ en 24 horas, $y$ luego de la fermentación con $S$. cerevisiae, obtuvieron cuatro muestras de bioetanol, que al mezclarse produjo una muestra de $95 \mathrm{ml}$ de solución con una concentración de alcohol al $27 \%$ v/v $(4,70 \mathrm{~g} / 100 \mathrm{~g})$.

Los rendimientos estequiométricos de AzR y etanol celulósico fueron calculados a partir de los datos obtenidos experimentalmente y se muestran en la siguiente tabla. 
Tabla 4.

Rendimiento estequiométrico de la cáscara de maní

\begin{tabular}{|c|c|c|c|c|}
\hline AzR & Etanol dest. & $\begin{array}{c}\text { Yp/s } \\
\text { exp. }\end{array}$ & $\begin{array}{c}\mathbf{Y x} / \mathbf{s} \\
\text { exp. }\end{array}$ & $\begin{array}{c}\mathbf{C O}_{2} \\
\text { calculado }\end{array}$ \\
\hline $\begin{array}{c}6,1767 \\
\text { g/20 g de Cáscara } \\
\text { de Maní }\end{array}$ & $\begin{array}{c}0,2828 \\
\text { g/20 g de } \\
\text { Cáscara de Maní }\end{array}$ & $\begin{array}{c}0,0458 \mathrm{~g} / \mathrm{g} \\
\mathrm{AzR}\end{array}$ & $\begin{array}{c}3,87 \mathrm{~g} / \mathrm{g} \\
\mathrm{AzR}\end{array}$ & $\begin{array}{c}0,64 \mathrm{~g} / \mathrm{g} \\
\mathrm{AzR}\end{array}$ \\
\hline
\end{tabular}

$A z R_{1}$ azúcares reductores iniciales, Yp/s: rendimiento de producto respecto al sustrato, $Y x / s$ : rendimiento de biomasa respecto al sustrato.

\section{Conclusión}

Los procesos de acondicionamiento y pretratamiento para residuos estudiados resultaron ser además de sencillos, esenciales al momento de realizar la hidrólisis, ya que las condiciones normales de los residuos, dificultaban el procesamiento y el almacenamiento de los mismos, por su porcentaje de humedad y tamaño de partícula; por otra parte la reducción del tamaño de partícula, facilitó la disolución del residuo en el medio básico durante la hidrólisis.

Las pruebas de múltiples rangos demostraron que las diferentes concentraciones de $\mathrm{NaOH}$ aplicadas en el pretratamiento hidrolítico básico no tuvieron una influencia estadística significativa en el rendimiento de AzR producido, mientras que la temperatura fue clave en el proceso de hidrólisis; esto influyó significativamente al rendimiento de $A z R$ producido, los rendimientos máximos de $A z R$ se obtuvieron a la temperatura más alta. Los cálculos de los coeficientes estequiométricos se realizaron a partir del rendimiento experimental, el cual fue 0,0458 gramos de etanol por gramo de AzR producido, a partir de la Cáscara de Maní; lo cual indica que el presente trabajo se encuentra dentro de los límites establecidos para la obtención de bioetanol, en comparación con los resultados obtenidos en las investigaciones antes mencionadas. Esto sin tener en cuenta que no se utilizaron métodos para optimizar la fermentación. Teniendo en cuenta esto y utilizando el residuo adecuado, la presente investigación propone un método de producción de bioetanol a partir de la hidrólisis la cascarilla de Maní. 


\section{Bibliografía}

Alam, J. B., Wadud, Z., Alam, J. B., \& Polak, J. W. (2013). Energy demand and economic consequences of transport policy. International Journal of Environmental Science and Technology, 10(5), 10751082.

Álvarez, A., García, E., Domínguez, M. M., Granandos, J. M., Aguirre, A., Carmona, R., Morales, A., Herrera, P. J., Licea, A., \& Mendoza, A. M. (2012). Aprovechamiento integral de los materiales lignocelulósicos. 11.

Amestoy Alonzo, J. (2010). El Planeta Tierra en peligro: Calentamiento Global, Cambio Climático (Vol. 4). Club Universal.

Bakare, V., Abdulsalami, M. S., Onusiriuka, B. C., Appah, J., Benjamin, B., \& Ndibe, T. O. (2019). Ethanol production from lignocellulosic materials by fermentation process using yeast. Journal of Applied Sciences and Environmental Management, 23(5), 875. https://doi.org/10.4314/jasem. v23i5.17

Balat, M. (2007). Global Bio-Fuel Processing and Production Trends. Energy Exploration \& Exploitation, 25(3), 195-218.

Balat, M. (2011). Production of bioethanol from lignocellulosic materials via the biochemical pathway: A review. Energy Conversion and Management, 52(2), 858-875.

Balat, M., \& Balat, H. (2009). Recent trends in global production and utilization of bio-ethanol fuel. Applied Energy, 86(11), 2273-2282.

Barakat, A., Monlau, F., Solhy, A., \& Carrere, H. (2015). Mechanical dissociation and fragmentation of lignocellulosic biomass: Effect of initial moisture, biochemical and structural proprieties on energy requirement. Applied Energy, 142, 240-246.

Barakat, A., Putaux, J.-L., Saulnier, L., Chabbert, B., \& Cathala, B. (2007). Characterization of Arabinoxylan-Dehydrogenati on Polymer (Synthetic Lignin Polymer) Nanoparticles. Biomacromolecules, 8(4), 1236-1245.

Bastidas, J. E. G. (2014). Diseño del proceso físico químico para la producción de azúcares reductores a partir del residuo de la extracción de aceite de piñón (Jatropha curcas). Escuela Politécnica Nacional.

Bello Gil, D., Carrera Bocour, E., \& Díaz Maqueira, Y. (2006, agosto). Determinación de azúcares reductores totales en jugos mezclados de caña de azúcar utilizando el método del ácido 3,5 dinitrosalicílico. 40(2), 45-50. 
Cabrera, E., Muñoz, M. J., Martín, R., Caro, I., Curbelo, C., \& Díaz, A. B. (2014). Alkaline and alkaline peroxide pretreatments at mild temperature to enhance enzymatic hydrolysis of rice hulls and straw. Bioresource Technology, 167, 1-7.

Cacua, A., Gelvez, J. J., Rodríguez, D. C., \& Parra, J. W. (2018). Production of bioethanol from rice husk pretreated with alkalis and hydrolyzed with acid cellulase at pilot scale. Journal of Physics: Conference Series, 1126, 1234.

Camacho Villanueva, E. A., García Pérez, J. A., Gutiérrez Rivera, B., \& Alarcón Gutiérrez, E. (2015). Pretratamiento Químico e hidrólisis enzimática de pulpa de café para obtención de azúcares reductores totales. II Reunión nacional de la red temática de bioenergía, 1. Cartel.

Chávez Porras, Á., \& Rodríguez González, A. (2016). Aprovechamiento de residuos orgánicos agrícolas y forestales en lberoamérica. Academia y Virtualidad, 9(2), 90-107.

Doran, P. M. (1998). Bioprocess Engineering Principles (Vol. 1). Acribia S.A.
El Comercio. (2018, Agosto 6). Ecopaís es la gasolina con más demanda.

El Comercio. (2019, Septiembre 13). El Gobierno propone incluir más bioetanol en las gasolinas del país.

Escalante Hernández, H., Orduz Prada, J., Zapata Lesmes, $\mathrm{H}$. J., \& Cardona Ruiz, M. C. (2010). Atlas del potencial energético de la biomasa residual en Colombia. Unidad de Planeación Minero Energética: Instituto de Hidrología, Meteorología y Estudios Ambientales: Departamento Administrativo de Ciencia, Tecnología e Innovación Colciencias: Universidad Industrial de Santander.

Frei, C., Whitney, R., Schiffer, H.-W., Karl, R., Qahtani, A., Thomas, P., Turton, H., Densing, M., Panos, E., Volkart, K., \& Reiser, D. A. (2013). World Energy Scenarios: Composing energy futures to 2050(9). Report.

Galbe, M., \& Zacchi, G. (2007). Pretreatment of Lignocellulosic Materials for Efficient Bioethanol Production. Biofuels, 108, 4165.

Griffiths, N. A., Rau, B. M., Vaché, K. B., Starr, G., Bitew, M. M., Aubrey, D. P., Martin, J. A., Benton, E., \& Jackson, C. R. (2019). Environmental effects 
of short-rotation woody crops for bioenergy: What is and isn't known. GCB Bioenergy, 11(4), 554-572.

Guerrero, V. H., Endara, D., \& Rosas Laverde, R. M. (2015). Obtención de Carbón Activado a partir de Residuos Lignocelulósicos de Canelo, Laurel y Eucalipto. 36.

INEC. (2018). Instituto Nacional de Estadísticas y Censos. QuitoEcuador.

INER. (2016). Análisis de oportunidades de investigación, desarrollo e innovación en eficiencia energética y energías renovables en Ecuador (Quito).

Kim, I., \& Han, J. (2012). Optimization of alkaline pretreatment conditions for enhancing glucose yield of rice straw by response surface methodology. Biomass and Bioenergy, 46, 210-217.

Loboguerrero, A. C. (2007). Exploración de la factibilidad del aprovechamiento de los residuos de la extracción de aceite de palma para convertirlos en Azucares Fermentables (Universidad de los Andes. Facultad de Ingeniería).

https://repositorio.uniandes.e du.co/bitstream/handle/1992/ 23310/u295687.pdf?sequenc $\mathrm{e}=1$ \&isAllowed $=y$
McKendry, P. (2002). Energy production from biomass (part 1): overview of biomass. Bioresource technology, 83(1), 37-46. https://doi.org/10.1016/S0960 $\underline{-8524(01) 00118-3}$

Michelena, G., Bueno, G., Eng, F., Viñals, M., Álvarez, A., Bell, A., Carrera, E., \& Manganelly, E. (2009). Etanol celulósico una alternativa energética. Instituto Cubano de Investigaciones de los Derivados de la Caña de Azúcar (ICIDCA), 1, 10-16.

Moya, R., Tenorio, C., \& Oporto, G. (2019). Short Rotation Wood Crops in Latin American: A Review on Status and Potential Uses as Biofuel. Energies, 12(4), 705.

Nzelibe, H., \& Okafoagu, C. (2007). Optimization of ethanol production from Garcinia kola (bitter kola) pulp agrowaste. African Journal of Biotechnology, 6(17), 6.

Piñeros, Y., \& Otálvaro, A. M. (2010). Evaluación de la producción de etanol a partir de cascarilla de arroz pre-tratada con $\mathrm{NaOCl}$, mediante hidrólisis y fermentación simultáneas. 26-61.

Rodríguez, A. Á., García, C. P., \& Díaz, M. B. F. (2012). Caracterización química de biomasa y su relación con el poder calorífico. 12. 
Rodríguez, M. D., Castrillo, M. L., Velázquez, J. E., Kramer, G. R., Sedler, C., Zapata, P. D., \& Villalba, L. (2017). Obtención de azúcares fermentables a partir de aserrín de pino pretratado secuencialmente con ácidobase. Revista Internacional de Contaminación Ambiental, 33(2), 317-324.

Román-Collado, R., \& MoralesCarrión, A. V. (2018). Towards a sustainable growth in Latin America: A multiregional spatial decomposition analysis of the driving forces behind $\mathrm{CO} 2$ emissions changes. Energy Policy, 115, 273-280.
Saval, S. (2012). Aprovechamiento de Residuos Agroindustriales: Pasado, Presente y Futuro. 16(2), 33.

Sierra, S. C., Chavarriaga, P., \& López, C. (2010). Biocombustibles y biotecnología: la yuca (manihot esculenta) como modelo de investigación. 22.

Torres Robles, R., Cano López, M. N., \& Aburto Anell, J. (2018). Obtención de bioetanol a partir de pasto kikuyo. 5(3), 23.

Yacobucci, B. D., \& Schnepf, R. (2007). Infrastructure, and Market Constraints Related to Expanded Production. 16. 